\title{
On the $L^{2}$-norm of Gegenbauer polynomials
}

\author{
Damir Ferizović ${ }^{1}$ (])
}

Received: 19 October 2020 / Accepted: 2 April 2021 / Published online: 21 April 2021

(c) The Author(s) 2021

\begin{abstract}
Gegenbauer, also known as ultra-spherical, polynomials appear often in numerical analysis or interpolation. In the present text we find a recursive formula for and compute the asymptotic behavior of their $L^{2}$-norm.
\end{abstract}

Keywords Gegenbauer polynomials $\cdot L^{2}$-norm $\cdot$ Asymptotics

Mathematics Subject Classification $33 \mathrm{C} 45 \cdot 33 \mathrm{~F} 99 \cdot 41 \mathrm{~A} 60$

\section{Introduction}

Gegenbauer polynomials $\mathcal{C}_{n}^{(\lambda)}, \quad$ where $\lambda \in I_{G}:=\left(-\frac{1}{2}, 0\right) \cup(0, \infty)$ is called the index and $n \in \mathbb{N}_{0}$ is the degree, are the coefficients of following power series expansion in $\alpha$ :

$\left(1-2 x \alpha+\alpha^{2}\right)^{-\lambda}=\sum_{n=0}^{\infty} \mathcal{C}_{n}^{(\lambda)}(x) \alpha^{n}$.

The case $\lambda=0$ is not considered here. $\left\{\mathcal{C}_{n}^{(\lambda)}\right\}_{n \in \mathbb{N}_{0}}$ are orthogonal with respect to the measure $\left(1-x^{2}\right)^{\lambda-1 / 2} \mathrm{~d} x$ over $[-1,1]$, and by [4] [Eq. 8.930]:

$\forall \lambda \in I_{G}: \mathcal{C}_{0}^{(\lambda)}(x)=1, \mathcal{C}_{1}^{(\lambda)}(x)=2 \lambda x$.

In the tables [4] by Gradshteyn and Ryzhik five pages are devoted to various integrals of Gegenbauer polynomials, for they appear in many branches of mathematics and theoretical physics, but not all cases of interest are covered: in applications to meson physics in [5], integrals of the type

The author thankfully acknowledges support by the Austrian Science Fund (FWF): F5503 "Quasi-Monte Carlo Methods" and FWF: W1230 "Doctoral School Discrete Mathematics," and the Austrian Marshall Plan Foundation.

Damir Ferizović

damir.ferizovic@tugraz.at

1 Graz University of Technology Institute of Analysis and Number Theory, Kopernikusgasse 24/II, 8010 Graz, Austria
$\int_{-1}^{1}\left[\mathcal{C}_{\ell}^{(\alpha)}(x)\right]^{2}\left(1-x^{2}\right)^{\alpha-3 / 2-p} \mathrm{~d} x$

had to be evaluated for $\ell \in \mathbb{N}_{0}, p \in \mathbb{Z}$ and $\operatorname{Re}\left(\alpha-\frac{1}{2}-p\right)>0$, where the notation follows [6]. Later, Rashid obtained in [6] a general identity for

$\int_{-1}^{1} \mathcal{C}_{\ell}^{(\alpha)}(x) \mathcal{C}_{k}^{(\beta)}(x)\left(1-x^{2}\right)^{\frac{\alpha+\beta-3}{2}-p} \mathrm{~d} x$,

in terms of a hyper-geometric series $F_{3}$ evaluated at 1 for $\ell, k \in \mathbb{N}_{0}, p \in \mathbb{Z}$ and $\operatorname{Re}((\alpha+\beta-1) / 2-p)>0$. Due to the close connection of zonal harmonics to Gegenbauer polynomials, similar integrals also appear in applications of determinantal point processes to energy estimates on spheres [2]. Yet the basic question of the $L^{2}$-norm of these polynomials has not been addressed in the literature, and we will fill this gap. The following notation will be used:

$\|f\|_{2}^{2}:=\int_{0}^{1}[f(x)]^{2} \mathrm{~d} x$.

We derive an asymptotic formula for $\left\|\mathcal{C}_{n}^{(\lambda)}\right\|_{2}^{2}$ when $\lambda>0$ in Proposition 1. Indeed, one of the key ingredients in [1] was the asymptotic nature of $\left\|\mathcal{C}_{n}^{(2)}\right\|_{2}^{2}$ in $n$, and the following lemma was proved in [1][Lemmas 6.1 and 6.2]:

Lemma 1 Let $\psi$ denote the digamma function and $\gamma$ the Euler-Mascheroni constant. Then the Gegenbauer polynomials satisfy for $n \geq 2$ : 


$$
\begin{aligned}
\left\|\sqrt{1-x^{2}} \mathcal{C}_{n-2}^{(2)}\right\|_{2}^{2}= & \frac{1}{16}\left(2 n^{2}-1\right)\left(\psi\left(n+\frac{1}{2}\right)+\gamma+\log (4)\right)-\frac{1}{8} n^{2}, \\
\left\|\mathcal{C}_{n-2}^{(2)}\right\|_{2}^{2}= & \frac{1}{16} n^{4}+\frac{1}{64}\left(4 n^{2}-1\right) \\
& \left(\psi\left(n+\frac{1}{2}\right)+\gamma+\log (4)\right)-\frac{5}{32} n^{2} .
\end{aligned}
$$

The following result of Corollary 5.2 from [3] will prove to be indispensable.

Theorem 1 (Dette [3]) The Gegenbauer polynomials satisfy for $\lambda \in I_{G}$

$$
\begin{aligned}
& \left(\frac{n}{2 \lambda}\right)^{2}\left[\mathcal{C}_{n}^{(\lambda)}(x)\right]^{2}+\left(1-x^{2}\right)\left[\mathcal{C}_{n-1}^{(\lambda+1)}(x)\right]^{2} \\
& =\sum_{k=0}^{n-1} \frac{\lambda+k}{\lambda}\left[\mathcal{C}_{k}^{(\lambda)}(x)\right]^{2} .
\end{aligned}
$$

Our main theorem is as follows, and we will use it to derive the asymptotic behavior of $\left\|\mathcal{C}_{n}^{(\lambda)}\right\|_{2}^{2}$.

Theorem 2 (Main Result) The Gegenbauer polynomials satisfy for $\lambda \in I_{G}$ :

$$
\begin{aligned}
& \left\|\mathcal{C}_{n-2}^{(\lambda+1)}\right\|_{2}^{2}=\frac{n^{2}-2 \lambda n}{2^{4} \lambda^{3}}\left[\mathcal{C}_{n}^{(\lambda)}(1)\right]^{2} \\
& \quad+\frac{n(2 n+1)}{2^{3} \lambda^{2}}\left\|\mathcal{C}_{n}^{(\lambda)}\right\|_{2}^{2}-\sum_{k=0}^{n-1} \frac{\lambda+k}{2^{2} \lambda^{2}}\left\|\mathcal{C}_{k}^{(\lambda)}\right\|_{2}^{2} .
\end{aligned}
$$

From Theorem 2 we can deduce following more compact formulas.

Corollary 1 The Gegenbauer polynomials satisfy for $\lambda \in I_{G}$ and $n>0$ :

$$
\begin{aligned}
\| & \sqrt{1-x^{2}} \mathcal{C}_{n-1}^{(\lambda+1)} \|_{2}^{2}=\left[\mathcal{C}_{n}^{(\lambda)}(1)\right]^{2} \frac{n+2 \lambda}{n+1} \frac{1-2 \lambda}{2^{3} \lambda^{2}} \\
& +\frac{(n+1)(2 n+3)}{2^{3} \lambda^{2}}\left\|\mathcal{C}_{n+1}^{(\lambda)}\right\|_{2}^{2}-\left\|\mathcal{C}_{n}^{(\lambda)}\right\|_{2}^{2} \frac{n+2 \lambda}{2^{3} \lambda^{2}} .
\end{aligned}
$$

Corollary 2 The Gegenbauer polynomials satisfy for $\lambda \in I_{G}$ and $n>1$ :

$$
\begin{array}{r}
\left\|\mathcal{C}_{n-2}^{(\lambda+1)}\right\|_{2}^{2}=\frac{n^{2}-2 \lambda n}{2^{4} \lambda^{3}}\left[\mathcal{C}_{n}^{(\lambda)}(1)\right]^{2} \\
+\frac{2 n^{2}(4 \lambda-1)+n(4 \lambda+1)+2 \lambda}{2^{5} \lambda^{3}}\left\|\mathcal{C}_{n}^{(\lambda)}\right\|_{2}^{2} \\
-\left[\mathcal{C}_{n}^{(\lambda)}(1)\right]^{2} \frac{n+2 \lambda}{n+1} \frac{1-2 \lambda}{2^{5} \lambda^{3}}-\frac{(n+1)(2 n+3)}{2^{5} \lambda^{3}}\left\|\mathcal{C}_{n+1}^{(\lambda)}\right\|_{2}^{2} .
\end{array}
$$

Proposition 1 Let $\mathcal{B}(x, y)$ denote the beta function. The following asymptotic formulas in $n$ hold for $\lambda \in(0,1)$ and $\delta=\max \{4 \lambda-1,2 \lambda\}$ :

$$
\begin{aligned}
\left\|\mathcal{C}_{n}^{(\lambda)}\right\|_{2}^{2} & <\mathcal{B}\left(1-\lambda, \frac{1}{2}\right) \frac{2^{1-2 \lambda}}{\Gamma(\lambda)^{2}} \frac{1}{n^{2-2 \lambda}}, \\
\left\|\mathcal{C}_{n}^{(\lambda+1)}\right\|_{2}^{2} & =\frac{n^{4 \lambda}}{4 \lambda \Gamma(2 \lambda+1)^{2}}+O\left(n^{\delta}\right), \\
\left\|\sqrt{1-x^{2}} \mathcal{C}_{n-1}^{(\lambda+1)}\right\|_{2}^{2} & <\frac{\mathcal{B}\left(1-\lambda, \frac{1}{2}\right)}{\Gamma(\lambda+1)^{2}} \frac{n^{2 \lambda}}{2^{1+2 \lambda}}+O\left(n^{\delta-1}\right) .
\end{aligned}
$$

The following asymptotic formulas hold for $\lambda>1$ and $\rho=\max \{4 \lambda-3,2 \lambda\}$ :

$$
\begin{gathered}
\left\|\mathcal{C}_{n-2}^{(\lambda+1)}\right\|_{2}^{2}=\frac{n^{4 \lambda}}{4 \lambda \Gamma(2 \lambda+1)^{2}}+\frac{\lambda-1}{\Gamma(2 \lambda+1)^{2}} n^{4 \lambda-1}+O\left(n^{4 \lambda-2}\right), \\
\left\|\sqrt{1-x^{2}} \mathcal{C}_{n-1}^{(\lambda+1)}\right\|_{2}^{2}=\frac{2 \lambda-1}{4(\lambda-1) \Gamma(2 \lambda+1)^{2}} n^{4 \lambda-2}+O\left(n^{\rho}\right) .
\end{gathered}
$$

The identity $2 \cdot\left\|\mathcal{C}_{n}^{(1)}\right\|_{2}^{2}=\psi\left(n+\frac{3}{2}\right)+\gamma+\log (4)$ is given in [1][Eq. 14].

\section{Ingredients for the Proof of the Theorem}

In this section we collect known results concerning Gegenbauer polynomials for later reference and the reader's convenience, and we derive some technical lemmas in Subsect. 2.1 to prove Theorem 2. To avoid repetition, we will assume $\lambda \in I_{G}$ for the rest of the text if not stated otherwise. Note first that

$$
\begin{aligned}
\frac{\mathrm{d}}{\mathrm{d} x} \mathcal{C}_{n+1}^{(\lambda)}(x) & =2 \lambda \mathcal{C}_{n}^{(\lambda+1)}(x) \quad \text { [4, Eq. 8.935], } \\
\mathcal{C}_{n}^{(\lambda)}(1) & =\frac{\Gamma(n+2 \lambda)}{\Gamma(2 \lambda) n !}=\frac{\prod_{j=1}^{n}(2 \lambda+n-j)}{n !} \text { [4, Eq. 8.937]; }
\end{aligned}
$$

and $\mathcal{C}_{n}^{(\lambda)}(1)$ is the maximum on $[-1,1]$ for $\lambda>0$ by [7] [Eq. 7.33.1]. Also, by (4):

$$
(n+2) \mathcal{C}_{n+2}^{(\lambda)}(x)=2 \lambda\left(x \mathcal{C}_{n+1}^{(\lambda+1)}(x)-\mathcal{C}_{n}^{(\lambda+1)}(x)\right) \quad \text { [4, Eq. 8.933.2], }
$$

$(n+\lambda) \mathcal{C}_{n}^{(\lambda)}(x)=\lambda\left(\mathcal{C}_{n}^{(\lambda+1)}(x)-\mathcal{C}_{n-2}^{(\lambda+1)}(x)\right)$

[4, Eq. 8.939.6].

\section{Identities for Gegenbauer polynomials}

Lemma 2 The Gegenbauer polynomials satisfy following identities: 


$$
\begin{gathered}
\mathcal{C}_{n}^{(\lambda+1)}(x)+\mathcal{C}_{n-2}^{(\lambda+1)}(x)=2 x \mathcal{C}_{n-1}^{(\lambda+1)}(x)+\mathcal{C}_{n}^{(\lambda)}(x),(\star) \\
\int_{0}^{1}\left[\mathcal{C}_{n}^{(\lambda+1)}(x)\right]^{2}-\left[\mathcal{C}_{n-2}^{(\lambda+1)}(x)\right]^{2} \mathrm{~d} x \\
=\frac{n+\lambda}{2 \lambda^{2}}\left(\left[\mathcal{C}_{n}^{(\lambda)}(1)\right]^{2}+(2 \lambda-1)\left\|\mathcal{C}_{n}^{(\lambda)}\right\|_{2}^{2}\right) .
\end{gathered}
$$

Proof First we use (6) and apply (5) to the right-hand side below proving $(\star)$ while using the short-hand $\ell:=\lambda+1$ :

$$
\begin{aligned}
& \mathcal{C}_{n}^{(\ell)}(x)+\mathcal{C}_{n-2}^{(\ell)}(x)=\frac{n+\lambda}{\lambda} \mathcal{C}_{n}^{(\lambda)}(x) \\
& \quad+2 x \mathcal{C}_{n-1}^{(\ell)}(x)-\left(2 x \mathcal{C}_{n-1}^{(\ell)}(x)-2 \mathcal{C}_{n-2}^{(\ell)}(x)\right) .
\end{aligned}
$$

Next we obtain by the binomial theorem with (6), ( $\star$ ) and (4)

$$
\begin{aligned}
& {\left[\mathcal{C}_{n}^{(\lambda+1)}(x)\right]^{2}-\left[\mathcal{C}_{n-2}^{(\lambda+1)}(x)\right]^{2}} \\
& \quad=\frac{n+\lambda}{\lambda} \mathcal{C}_{n}^{(\lambda)}(x)\left(2 x \mathcal{C}_{n-1}^{(\lambda+1)}(x)+\mathcal{C}_{n}^{(\lambda)}(x)\right) \\
& \quad=\frac{n+\lambda}{\lambda}\left(\frac{x}{2 \lambda} \frac{\mathrm{d}}{\mathrm{d} x}\left[\mathcal{C}_{n}^{(\lambda)}(x)\right]^{2}+\left[\mathcal{C}_{n}^{(\lambda)}(x)\right]^{2}\right) .
\end{aligned}
$$

Integration by parts then finishes the argument.

Lemma 3 The Gegenbauer polynomials satisfy the following identity:

$$
\begin{aligned}
& \int_{0}^{1} x^{2}\left[\mathcal{C}_{n+1}^{(\lambda+1)}(x)\right]^{2}+\left[\mathcal{C}_{n}^{(\lambda+1)}(x)\right]^{2} \mathrm{~d} x \\
& +\frac{1}{2 \lambda} \int_{0}^{1}\left(1-x^{2}\right)\left[\mathcal{C}_{n+1}^{(\lambda+1)}(x)\right]^{2} \mathrm{~d} x \\
& \quad=\frac{(n+2)^{2}}{8 \lambda^{3}}\left[\mathcal{C}_{n+2}^{(\lambda)}(1)\right]^{2}+\frac{2 \lambda-1}{2 \lambda} \frac{(n+2)^{2}}{4 \lambda^{2}}\left\|\mathcal{C}_{n+2}^{(\lambda)}\right\|_{2}^{2} .
\end{aligned}
$$

Proof Let $n=2 m$. By Lemma 2 and a telescoping sum argument:

$$
\begin{aligned}
& \left\|\mathcal{C}_{n}^{(\lambda+1)}\right\|_{2}^{2}-\left\|\mathcal{C}_{0}^{(\lambda+1)}\right\|_{2}^{2} \\
& =\sum_{j=1}^{m} \frac{2 j+\lambda}{2 \lambda^{2}}\left(\left[\mathcal{C}_{2 j}^{(\lambda)}(1)\right]^{2}+(2 \lambda-1)\left\|\mathcal{C}_{2 j}^{(\lambda)}\right\|_{2}^{2}\right), \\
& \left\|\mathcal{C}_{n+1}^{(\lambda+1)}\right\|_{2}^{2}-\left\|\mathcal{C}_{1}^{(\lambda+1)}\right\|_{2}^{2} \\
& =\sum_{j=1}^{m} \frac{2 j+1+\lambda}{2 \lambda^{2}}\left(\left[\mathcal{C}_{2 j+1}^{(\lambda)}(1)\right]^{2}+(2 \lambda-1)\left\|\mathcal{C}_{2 j+1}^{(\lambda)}\right\|_{2}^{2}\right) .
\end{aligned}
$$

Using (1) and summing up, and an application of Dette's result (2) yields:

$$
\begin{aligned}
\int_{0}^{1} & {\left[\mathcal{C}_{n+1}^{(\lambda+1)}(x)\right]^{2}+\left[\mathcal{C}_{n}^{(\lambda+1)}(x)\right]^{2} \mathrm{~d} x } \\
= & \frac{4}{3}(\lambda+1)^{2}+1+\frac{1}{2 \lambda} \sum_{j=2}^{n+1} \frac{j+\lambda}{\lambda}\left[\mathcal{C}_{j}^{(\lambda)}(1)\right]^{2} \\
& +\frac{2 \lambda-1}{2 \lambda} \sum_{j=2}^{n+1} \frac{j+\lambda}{\lambda}\left\|\mathcal{C}_{j}^{(\lambda)}\right\|_{2}^{2} \\
= & \frac{(n+2)^{2}}{8 \lambda^{3}}\left[\mathcal{C}_{n+2}^{(\lambda)}(1)\right]^{2} \\
& +\frac{2 \lambda-1}{2 \lambda} \sum_{j=0}^{n+1} \frac{j+\lambda}{\lambda}\left\|\mathcal{C}_{j}^{(\lambda)}\right\|_{2}^{2} \\
= & \frac{(n+2)^{2}}{8 \lambda^{3}}\left[\mathcal{C}_{n+2}^{(\lambda)}(1)\right]^{2} \\
& +\frac{2 \lambda-1}{2 \lambda}\left(\frac{(n+2)^{2}}{4 \lambda^{2}}\left\|\mathcal{C}_{n+2}^{(\lambda)}\right\|_{2}^{2}+\left\|\sqrt{1-x^{2}} \mathcal{C}_{n+1}^{(\lambda+1)}\right\|_{2}^{2}\right) .
\end{aligned}
$$

The case $n+1=2 m$ is analogous.

Lemma 4 The Gegenbauer polynomials satisfy the following identity:

$$
\begin{aligned}
& \int_{0}^{1} x^{2}\left[\mathcal{C}_{n+1}^{(\lambda+1)}(x)\right]^{2}-\left[\mathcal{C}_{n}^{(\lambda+1)}(x)\right]^{2} \mathrm{~d} x \\
& =\frac{n+2}{4 \lambda^{2}}\left(\left[\mathcal{C}_{n+2}^{(\lambda)}(1)\right]^{2}-(n+3)\left\|\mathcal{C}_{n+2}^{(\lambda)}\right\|_{2}^{2}\right) .
\end{aligned}
$$

Proof Note first that by (5) and by quadratic completion

$$
\begin{aligned}
2 \frac{n+2}{2 \lambda} \mathcal{C}_{n+2}^{(\lambda)}(x) \mathcal{C}_{n}^{(\lambda+1)}(x) \\
=2 x \mathcal{C}_{n+1}^{(\lambda+1)}(x) \mathcal{C}_{n}^{(\lambda+1)}(x)-2\left[\mathcal{C}_{n}^{(\lambda+1)}(x)\right]^{2} \\
=x^{2}\left[\mathcal{C}_{n+1}^{(\lambda+1)}(x)\right]^{2}-\left[\mathcal{C}_{n}^{(\lambda+1)}(x)\right]^{2} \\
\quad-\left(x \mathcal{C}_{n+1}^{(\lambda+1)}(x)-\mathcal{C}_{n}^{(\lambda+1)}(x)\right)^{2}
\end{aligned}
$$

Hence by the binomial theorem and again by (5)

$$
\begin{aligned}
& 2 \int_{0}^{1} x^{2}\left[\mathcal{C}_{n+1}^{(\lambda+1)}(x)\right]^{2}-\left[\mathcal{C}_{n}^{(\lambda+1)}(x)\right]^{2} \mathrm{~d} x \\
& =\frac{n+2}{\lambda} \int_{0}^{1}\left(x \mathcal{C}_{n+1}^{(\lambda+1)}(x)+\mathcal{C}_{n}^{(\lambda+1)}(x)\right) \mathcal{C}_{n+2}^{(\lambda)}(x) \mathrm{d} x \\
& =\frac{n+2}{\lambda} \int_{0}^{1} \frac{x}{4 \lambda} \frac{\mathrm{d}}{\mathrm{d} x}\left[\mathcal{C}_{n+2}^{(\lambda)}(x)\right]^{2}+\mathcal{C}_{n}^{(\lambda+1)}(x) \mathcal{C}_{n+2}^{(\lambda)}(x) \mathrm{d} x \\
& =\frac{n+2}{4 \lambda^{2}}\left(\left[\mathcal{C}_{n+2}^{(\lambda)}(1)\right]^{2}-\int_{0}^{1}\left[\mathcal{C}_{n+2}^{(\lambda)}(x)\right]^{2} \mathrm{~d} x\right) \\
& \quad+2 \frac{n+2}{2 \lambda} \int_{0}^{1} \mathcal{C}_{n}^{(\lambda+1)}(x) \mathcal{C}_{n+2}^{(\lambda)}(x) \mathrm{d} x
\end{aligned}
$$

which proves the result when we substitute (7) and use (5) one last time. 


\section{Proof of the main results}

Proof of Theorem 2 Subtract the left-hand sides of Lemma 3 and Lemma 4:

$$
\begin{aligned}
& 2\left\|\mathcal{C}_{n}^{(\lambda+1)}\right\|_{2}^{2}+\frac{1}{2 \lambda}\left\|\sqrt{1-x^{2}} \mathcal{C}_{n+1}^{(\lambda+1)}\right\|_{2}^{2} \\
& =\left(\frac{(n+2)^{2}}{8 \lambda^{3}}-\frac{n+2}{4 \lambda^{2}}\right)\left[\mathcal{C}_{n+2}^{(\lambda)}(1)\right]^{2} \\
& \quad+\left(\frac{(n+2)^{2}}{4 \lambda^{2}}+\frac{(n+2)(n+3)}{4 \lambda^{2}}\right)\left\|\mathcal{C}_{n+2}^{(\lambda)}\right\|_{2}^{2} \\
& \quad-\frac{1}{2 \lambda} \frac{(n+2)^{2}}{4 \lambda^{2}}\left\|\mathcal{C}_{n+2}^{(\lambda)}\right\|_{2}^{2} ;
\end{aligned}
$$

an application of Dette's formula (2) then gives the desired expression.

Proof of Corollary 1 We use Lemma 4, add zero and obtain with Theorem 2

$$
\begin{aligned}
& \frac{n}{4 \lambda^{2}}\left(\left[\mathcal{C}_{n}^{(\lambda)}(1)\right]^{2}-(n+1)\left\|\mathcal{C}_{n}^{(\lambda)}\right\|_{2}^{2}\right) \\
& +\int_{0}^{1}\left(1-x^{2}\right)\left[\mathcal{C}_{n-1}^{(\lambda+1)}(x)\right]^{2} \mathrm{~d} x \\
& =\int_{0}^{1}\left[\mathcal{C}_{n-1}^{(\lambda+1)}(x)\right]^{2}-\left[\mathcal{C}_{n-2}^{(\lambda+1)}(x)\right]^{2} \mathrm{~d} x \\
& =\frac{(n+1)^{2}-2 \lambda(n+1)}{2^{4} \lambda^{3}}\left[\mathcal{C}_{n+1}^{(\lambda)}(1)\right]^{2} \\
& +\frac{(n+1)(2 n+3)}{2^{3} \lambda^{2}}\left\|\mathcal{C}_{n+1}^{(\lambda)}\right\|_{2}^{2} \\
& -\sum_{k=0}^{n} \frac{\lambda+k}{2^{2} \lambda^{2}}\left\|\mathcal{C}_{k}^{(\lambda)}\right\|_{2}^{2} \\
& -\frac{n^{2}-2 \lambda n}{2^{4} \lambda^{3}}\left[\mathcal{C}_{n}^{(\lambda)}(1)\right]^{2}-\frac{n(2 n+1)}{2^{3} \lambda^{2}} \\
& \left\|\mathcal{C}_{n}^{(\lambda)}\right\|_{2}^{2}+\sum_{k=0}^{n-1} \frac{\lambda+k}{2^{2} \lambda^{2}}\left\|\mathcal{C}_{k}^{(\lambda)}\right\|_{2}^{2} \\
& =\left[\mathcal{C}_{n}^{(\lambda)}(1)\right]^{2} \\
& \left(\frac{(n+1)^{2}-2 \lambda(n+1)}{2^{4} \lambda^{3}} \frac{(n+2 \lambda)^{2}}{(n+1)^{2}}-\frac{n^{2}-2 \lambda n}{2^{4} \lambda^{3}}\right) \\
& +\frac{(n+1)(2 n+3)}{2^{3} \lambda^{2}}\left\|\mathcal{C}_{n+1}^{(\lambda)}\right\|_{2}^{2}-\left\|\mathcal{C}_{n}^{(\lambda)}\right\|_{2}^{2} \\
& \left(\frac{n(2 n+1)}{2^{3} \lambda^{2}}+\frac{\lambda+n}{2^{2} \lambda^{2}}\right) \\
& =\left[\mathcal{C}_{n}^{(\lambda)}(1)\right]^{2} \frac{2 n^{2}+3 n+2 \lambda-2 \lambda n-4 \lambda^{2}}{2^{3} \lambda^{2}(n+1)} \\
& +\frac{(n+1)(2 n+3)}{2^{3} \lambda^{2}}\left\|\mathcal{C}_{n+1}^{(\lambda)}\right\|_{2}^{2}-\left\|\mathcal{C}_{n}^{(\lambda)}\right\|_{2}^{2} \\
& \left(\frac{2 n^{2}+3 n+2 \lambda}{2^{3} \lambda^{2}}\right) \text {. }
\end{aligned}
$$

We re-order to obtain the result.
Proof of Corollary 2 This follows directly from the proofs of Theorem 2 and Corollary 1.

Remark 1 For our asymptotic analysis we will need the following identity, see [8]: For $|z| \rightarrow \infty$ and $\alpha, \beta \geq 0$ :

$$
\begin{aligned}
& \frac{\Gamma(z+\alpha)}{\Gamma(z+\beta)}=z^{\alpha-\beta} \\
& \quad\left(1+\frac{(\alpha-\beta)(\alpha+\beta-1)}{2 z}+O\left(|z|^{-2}\right)\right),
\end{aligned}
$$

we obtain by (4) for $\lambda>0$ :

$$
\begin{aligned}
& \Gamma(2 \lambda)^{2} \cdot\left[\mathcal{C}_{n}^{(\lambda)}(1)\right]^{2}=n^{4 \lambda-2} \\
& \quad+2 \lambda(2 \lambda-1) n^{4 \lambda-3}+O\left(n^{4 \lambda-4}\right) .
\end{aligned}
$$

Proof of Proposition 1 We will write $\left\|\mathcal{C}_{n}^{(\lambda)}\right\|_{2}^{2}=\Theta\left(n^{\Phi(\lambda)}\right)$ if there are some constants $c_{1}, c_{2}>0$ such that $c_{1} n^{\Phi(\lambda)} \leq\left\|\mathcal{C}_{n}^{(\lambda)}\right\|_{2}^{2} \leq c_{2} n^{\Phi(\lambda)}$ for all $n$ big enough. First we use (3) to show by induction that $\Phi(\lambda)$ exists for $\lambda>1$, and that $\left[\mathcal{C}_{n}^{(\lambda)}(1)\right]^{2}=\Theta\left(n^{\Phi(\lambda)+2}\right)$.

The case $\lambda=m \in \mathbb{N}_{>1}$ : Lemma 1 gives the result for $\lambda=2$, and if it holds for $m$, then with (3) and abuse of notation we have:

$$
\begin{gathered}
\left\|\mathcal{C}_{n-2}^{(m+1)}\right\|_{2}^{2}=n^{2} \Theta\left(n^{\Phi(m)+2}\right)+n^{2} \Theta\left(n^{\Phi(m)}\right) \\
+\Theta\left(n^{\Phi(m)+2}\right)+n^{2} \Theta\left(n^{\Phi(m)}\right)
\end{gathered}
$$

This proves the claim as it shows that $\left\|\mathcal{C}_{n}^{(m+1)}\right\|_{2}^{2}=\Theta\left(n^{\Phi(m)+4}\right)$, but by (4):

$$
\begin{aligned}
\mathcal{C}_{n}^{(\lambda+1)}(1) & =\frac{(2 \lambda+n+1)(2 \lambda+n)}{2 \lambda(2 \lambda+1)} \mathcal{C}_{n}^{(\lambda)}(1) \\
& =\Theta\left(n^{2} \mathcal{C}_{n}^{(\lambda)}(1)\right)
\end{aligned}
$$

which, when squared and $\lambda=m$, is of $\operatorname{order} \Phi(m)+6$.

The case $\lambda \in(m, m+1)$ for $m \in \mathbb{N}$ : For $\lambda \in(0,1)$ and $\theta \in[0, \pi]:$

$\sin (\theta)^{\lambda}\left|\mathcal{C}_{n}^{(\lambda)}(\cos (\theta))\right|<\frac{2^{1-\lambda}}{\Gamma(\lambda)} n^{\lambda-1}$ see [7, Eq. 7.33.5].

We square this inequality, multiply by $\sin (\theta)^{1-2 \lambda}$ and integrate:

$$
\begin{gathered}
\left\|\mathcal{C}_{n}^{(\lambda)}\right\|_{2}^{2}<\frac{2^{2-2 \lambda}}{\Gamma(\lambda)^{2}} n^{2 \lambda-2} \int_{0}^{\pi / 2} \sin (\theta)^{1-2 \lambda} \mathrm{d} \theta \\
=\mathcal{B}\left(1-\lambda, \frac{1}{2}\right) \frac{2^{1-2 \lambda}}{\Gamma(\lambda)^{2}} n^{2 \lambda-2} ;
\end{gathered}
$$

where we used a change of variables $\theta=\arcsin (x)$ and $\mathcal{B}(x, y)$ is the beta function. This in combination with (10) and (3) gives for $\delta=\max \{4 \lambda-1,2 \lambda\}$ : 
$\left\|\mathcal{C}_{n}^{(\lambda+1)}\right\|_{2}^{2}=\frac{n^{4 \lambda}}{2^{4} \lambda^{3} \Gamma(2 \lambda)^{2}}+O\left(n^{\delta}\right)$.

Thus for $\quad \lambda \in(0,1): \quad \Phi(\lambda+1)=4 \lambda, \quad$ and $\left[\mathcal{C}_{n}^{(\lambda+1)}(1)\right]^{2}=\Theta\left(n^{4 \lambda+2}\right)$ by (10), which finishes the case for the interval $(1,2)$, and we use induction with (3) and (11).

Hence, the two leading terms in the asymptotic form of $\left\|\mathcal{C}_{n}^{(\lambda+1)}\right\|_{2}^{2}$ are in the expansion of $\mathcal{C}_{n}^{(\lambda)}(1)$ when $\lambda>1$; using once more (10) and (3) yields

$$
\begin{gathered}
\frac{n^{2}-2 \lambda n}{2^{4} \lambda^{3}}\left[\mathcal{C}_{n}^{(\lambda)}(1)\right]^{2}=\frac{n^{4 \lambda}}{4 \lambda \Gamma(2 \lambda+1)^{2}} \\
+\frac{2 \lambda(2 \lambda-2)}{4 \lambda \Gamma(2 \lambda+1)^{2}} n^{4 \lambda-1}+O\left(n^{4 \lambda-2}\right) .
\end{gathered}
$$

The asymptotic of the rest term follows by (3), Equation (12) and induction for non-integer $\lambda \in \mathbb{R}_{>2}$ or else Lemma 1 and induction when $\lambda \in \mathbb{N}_{>2}$. These asymptotic formulas in combination with Corollary 1 and (10) will now finish the argument. We will only do the case $\lambda>1$, for the case $\lambda \in(0,1)$ is similar; let $\rho=\max \{4 \lambda-3,2 \lambda\}$ :

$$
\begin{aligned}
\| & \sqrt{1-x^{2}} \mathcal{C}_{n-1}^{(\lambda+1)} \|_{2}^{2}=\frac{1-2 \lambda}{2^{3} \lambda^{2}}\left[\mathcal{C}_{n}^{(\lambda)}(1)\right]^{2} \\
& +\frac{2 n^{2}}{2^{3} \lambda^{2}}\left\|\mathcal{C}_{n+1}^{(\lambda)}\right\|_{2}^{2}+O\left(n^{\rho}\right) \\
= & \frac{1-2 \lambda}{2^{3} \lambda^{2}} \frac{n^{4 \lambda-2}}{\Gamma(2 \lambda)^{2}}+\frac{2 n^{2}}{2^{3} \lambda^{2}} \frac{n^{4 \lambda-4}}{4(\lambda-1) \Gamma(2 \lambda-1)^{2}}+O\left(n^{\rho}\right) \\
= & \frac{n^{4 \lambda-2}}{2^{3} \lambda^{2} \Gamma(2 \lambda-1)^{2}}\left(\frac{1-2 \lambda}{(2 \lambda-1)^{2}}+\frac{1}{2(\lambda-1)}\right)+O\left(n^{\rho}\right) \\
= & \frac{n^{4 \lambda-2}}{2^{3} \lambda^{2} \Gamma(2 \lambda-1)^{2}} \frac{1}{(2 \lambda-1)(\lambda-1) 2}+O\left(n^{\rho}\right) .
\end{aligned}
$$

Remark 2 One can use Lemma 1, Corollary 1 and Corollary 2 to find exact formulas for $\left\|\sqrt{1-x^{2}} \mathcal{C}_{n}^{(m)}\right\|_{2}^{2}$ and $\left\|\mathcal{C}_{n}^{(m)}\right\|_{2}^{2}$ where $m \in \mathbb{N}_{>1}$.

Acknowledgements The help of J. Brauchart, P. Grabner and J. Thuswaldner is gratefully appreciated, who proof read the manuscript, made useful remarks on presentation, made me aware of reference [6] and suggested to generalize Proposition 1 from $\lambda \in \frac{1}{2} \mathbb{N}$ to $\lambda>0$. This paper is published open access thanks to the TU Graz Open Access Publishing Fund. I thank the anonymous reviewer for helpful remarks.
Funding Open access funding provided by Graz University of Technology.

\section{Declarations}

Conflict of interest The author declares that he has no conflict of interest.

Open Access This article is licensed under a Creative Commons Attribution 4.0 International License, which permits use, sharing, adaptation, distribution and reproduction in any medium or format, as long as you give appropriate credit to the original author(s) and the source, provide a link to the Creative Commons licence, and indicate if changes were made. The images or other third party material in this article are included in the article's Creative Commons licence, unless indicated otherwise in a credit line to the material. If material is not included in the article's Creative Commons licence and your intended use is not permitted by statutory regulation or exceeds the permitted use, you will need to obtain permission directly from the copyright holder. To view a copy of this licence, visit http://creativecommons.org/licenses/by/4.0/.

\section{References}

1. Beltrán, C., Ferizović, D.: Approximation to uniform distribution in SO(3). Constr. Approx. 52, 283-311 (2020)

2. Beltrán, C., Marzo, J., Ortega-Cerdà, J.: Energy and discrepancy of rotationally invariant determinantal point processes in high dimensional spheres. J. Complex. 37, 76-109 (2016)

3. Dette, H.: New identities for orthogonal polynomials on a compact interval. J. Math. Anal. Appl. 179, 547-573 (1993)

4. Gradshteyn, I.S., Ryzhik, I.M., Jeffrey, A., Zwillinger, D.: Table of Integrals, Series, and Products, 6th edn. Academic Press, Cambridge (2000)

5. Laursen, M.L., Mita, K.: Some integrals involving associated Legendre functions and Gegenbauer polynomials. J. Phys. A: Math. Gen. 14, 1065-1068 (1981)

6. Rashid, M.A.: Evaluation of integrals involving powers of $\left(1-x^{2}\right)$ and two associated Legendre functions or Gegenbauer polynomials. J. Phys. A: Math. Gen. 19, 2505-2512 (1986)

7. Szegö, G.: Orthogonal Polynomials, 4th edn. Amer. Math, Soc (1975)

8. Tricomi, F., Erdélyi, A.: The Asymptotic Expansion of a Ration of Gamma Functions. Pac. J. Math. 1(1), 133-142 (1951)

Publisher's Note Springer Nature remains neutral with regard to jurisdictional claims in published maps and institutional affiliations. 